\title{
Using hand-draw maps of residential neighbourhood to compute level of circularity and investigate its predictors
}

\author{
Carlos Siordia*, Claudia J. Coulton ${ }^{\mathrm{b}}$
}

a University of Pittsburgh, USA

$b$ Case Western Reserve University, USA

Investigating individuals' Hand-Drawn Residential Neighbourhood (HDRN) maps may help determine some of the basic geometrical properties found in cognitive maps of meaningful geographical spaces. The main objective was to empirically determine an optimal radius size for measuring environmental attributes of the $\mathrm{RN}$ when using circular spatial buffers. HDRN maps from the Making Connections study were explored using minimum bounded circles (MBC). Baseline data on 4,742 community-dwelling adults showed that $30 \%$ of people drew HDRN that included $>70 \%$ of the MBC. We recommend 0.25 miles as an optimum radius size when attempting to measure aspects of the RN. Multivariate linear regressions indicated lower levels of circularity for females, non-Latinoothers, for people living longer in their residential neighbourhood, and for large drawn polygons. In contrast, regression results indicated higher levels of circularity for nonLatino-blacks (compared to non-Latino-whites) and those with some college education (compared to those with a bachelors' degree and beyond). We conclude with advice on best practices for measuring environmental attributes of residential neighbourhoods when using circular spatial buffers.

Key Words: neighbourhood, circularity, environment, buffers, ArcGIS.

Article Info: Received: June 11, 2015; Revised: October 24, 2015; Accepted: November 14, 2015; Online: November 30, 2015.

\section{Introduction}

Thinkers, such as geographers and social scientists (Duncan, 1957), have long posited that attributes of the environment interact bi-directionally with charac-

\footnotetext{
* Corresponding author

Address: 130 N Bellfield, Pittsburgh, PA 1512, USA.

Phone: +1 2402761185 | Email: cas271@pitt.edu
} 
teristics of the individual to affect their economic and physical well-being. For example, research has found "place matters" for disparities in diabetes (Gaskin et al., 2014), tobacco use (Huh et al., 2014), amongst many others health outcomes. Efforts have begun to develop health interventions that focus on transforming attributes of the residential environment (Gavin et al., 2015). Social determinants of health (e.g., childhood poverty and access to health care) are partially shaped by the social structures (e.g., systemic discrimination) influencing the geographic distribution of human populations. Measuring social structures, as they are manifested in aspects of the environment, may help identify novel risk factors from social determinants of health. In our analysis, we used views and methods from sociology, geography, and epidemiology to delineate the intricacies of how individuals define the geographical boundaries of their residential neighbourhoods.

Evidence has culminated over decades to provide evidence that better health outcomes are associated with habitats that provide safe green spaces, affordable healthy food, and contain multiple amenities in land-use-mixed environments (Ellen, Mijanovich, \& Dillman, 2001; Rosso et al., 2011; Schaefer-McDaniel et al., 2010; Yen, Michael, \& Perdue, 2009). In recent decades, the presumption that place matters has risen to prominence as researchers across many disciplines seek ways to include measures of the environment in hierarchical- and geographically-aware regression models. As a result of demands in the 'research market place', measures of the environment, statistical techniques, geographically referenced data, and software have significantly advanced in recent decades. Our investigation contributes to health and place research by scientifically investigating the geographical properties of participant-defined residential neighbourhoods boundaries.

Despite the proliferation of research that includes measures of environmental exposures, some gaps remain between theory and methods. One important missing link has to do with the ecological concept of neighbourhood. The idea that meaningful geographical spaces (e.g., neighbourhoods) play a role in how humans interact with one another and their environment is valuable in promoting the epistemological stance that social phenomena have the ability to influence health. Advancing research on how exposures from the physical (e.g., atmospheric particulates), social (e.g., neighbourhood cohesion), and built (e.g., sidewalk availability) environments affect health demands advancing measurement methodologies through scientific methods. We hope our study helps in these efforts.

Existing gaps between theory and methods are driven by multiple factors including the limited availability of empirical evidence on the geographical properties of person-defined neighbourhood polygons. Our study is amongst the first to use a unique source of information to explore, through computational geometry, the level of circularity in Hand-Drawn Residential Neighbourhood (HDRN). Our investigation fills a detrimental gap in the literature by delineating the degree to which community-dwelling adults from multiple metropolitan cities in the United States (US) draw their HDRN as a circle. We sought to answer to research questions: (1) are circular spatial buffers representative of how individuals define the geographical boundaries of their residential neighbourhood?; (2) when using circular spatial buffers to measure 
environmental characteristics of the residential neighbourhood, what is the best radius size? We compliment these questions by showing how 'level of circularity' (defined below) varies as a function of basic demographic characteristics.

\section{Neighbourhood}

Publications using different spatial units to measure characteristics of place frequently use the term neighbourhood-unintentionally giving the illusion that homogeneous procedures are used between studies to measure neighbourhoods. Because the term neighbourhood is so widely used, entering the term in any academic search engine will produce thousands of manuscripts. The volume of publications using the word neighbourhood may erroneously lead nonexperts to conclude that a widely held definition of the term exists. In truth, standard procedures for operationalizing neighbourhood remain elusive. For example, the operationalization of neighbourhood in quantitative research within the US is primarily limited by available data-where a wide array of spatial administrative units (e.g., census blocks, tracts, zip codes, etc.) or various distance-based divisions limits scientific development. Because neighbourhood may be a rhetorically powerful term in public discourse, it is important that scientific evidence be used to standardize its measurement. If using place-based zones, such as circular spatial buffers anchored on place of residence, is deemed a superior methodological approach than using administrative spatial units, then providing guidance for best practices for circular spatial buffers is needed. Our discussion focuses on informing best practices when using circular spatial buffers to quantify aspects of the residential neighbourhood.

Measuring aspects of the neighbourhood is commonly seen as important because attributes in this physical space are hypothesized to play a causal role in an individual's behavior, health, and economic well-being (Newburger et al, 2011). Although the term neighbourhood may be laden with ambiguous scientific meaning, the social construct may be argued to have deep social meaning for many individuals (Siordia \& Saenz, 2013). As a result, the term neighbourhood can be used as a rhetorical instrument for advancing the views of an argument. For example, employing the term in manuscripts can make otherwise dense material into marketable products with an easy-to-understand message. Unfortunately, continuous reiteration of the term neighbourhood has made it difficult to highlight its ambiguity. The wide use of the term neighbourhood has promoted research while simultaneously obfuscating scientific knowledge. We hope our discussion contributes towards advancing its measurement through improved operationalization.

Despite the overwhelming amount of use the term neighbourhood receives in publications, challenges remain in how best to conceptualize and measure attributes of the residential environment. Fortunately, many researchers over the last decades have devoted great effort to explaining challenges associated with measuring the environment. For example, authors have discussed the Mapable Areal Unit Problem (MAUP) - the idea that measuring a particular attribute of place (e.g., percent in-poverty) with different geographical scales (e.g., state vs. county) can produce different results (Openshaw, 1984). Others have subsequently discussed the Uncertain Geographic Context Problem 
(UGeoCP) - the view that singular measures of the environment do not necessarily apply to the true exposures the individual experiences (Kwan, 2012a). More recently, authors have introduced the complex view of Spatial Polygamy and Contextual Exposures (SPACEs) - a rubric on how individuals experience their environments over a time and space continuum (Matthews \& Yang, 2013). While MAUP argues that the size of the polygon used to measure place matters, UGeoCP focuses on the true link between place attributes and the person, and SPACES highlights the fact that time-stamped and person-aware measures may be the way to advance research on person environment interaction. Our approach adds to previous work (James et al., 2014) showing that the shape of geographical polygons representing meaningful places matters when attempting to quantify environmental exposures. More simply, we argue that the properties of the geometrical forms used to capture a person's habitat merits special attention. Researchers should highlight the various assumptions with using alternate geometrical forms (e.g., circular or street networks buffers) to quantify exposures in the environment.

We term this challenge for measuring the environment as Searching Heterogeneity of Area and Perimeter in measures of the Environment (SHAPE). Unlike MAUP, UGeoCP, and SPACES, we focus on the idea that measuring environmental exposures through alternate geometrical shapes can produce different results. Discussing difficulties with measurements of environmental exposures should include awareness of SHAPE related challenges. In particular, researchers should acknowledge that imposed geometries (such as circles) may not properly reflect how individuals perceive or inhabit the geographical boundaries of what they deem as meaningful physical spaces.

Why should spatial buffers be considered a methodologically superior approach than using administrative geographical units? In the US, most geographically referenced studies produce measures of the social environment by using administrative spatial units such as US Census block, tract, or counties. These administrative units may be argued to be non-theory-driven. Their geographical characteristics (e.g., boundaries) are determined by the administrative needs of federal and local government agencies. The boundaries of these administrative spatial units are not influenced by a theory or conceptual framework on how humans interact with physical spaces over time. Because the majority of data on social environments is available with administrative units, they are frequently used in research. Unfortunately, using administrative spatial units simultaneously allows the production of research while unintentionally disguising the fact that neighbourhood is measured non-systematically between some studies.

Defining the geographical boundaries of neighbourhoods with administrative units probably means that: no definitive solution will be found for the MAUP; that UGeoCP will be likely be present in most measures of the environment; that issues with SPACES will rarely be acknowledged; and that special challenges from SHAPE will remain beyond scientific exploration. Because the fundamental idea that place matters has yet to be convincingly refuted, technical solutions and informally agreed upon procedures have helped establish protocols for how to produce measures of place that allow for inter-study comparisons (Coulton et al, 2004). Until more time- and place-specific measures of environmental 
exposures-through Global Positioning System (GPS) devices-are more readily available and easily consumable, promoting the establishment of measuring protocols that increase inter-study comparability could be beneficial for advancing health and place research.

As mentioned before, conceptual definitions and the operationalization of neighbourhood have both been limited by the availability of data resources (Coulton et al, 2001). A few studies have been undertaken to delineate the intricacies of what individuals perceive as their residential neighbourhood. For example, a study using hand-drawn maps of residential neighbourhoods from Making Connections data found that "residents living in close proximity to one another" had "a number of divergent opinions about neighbourhood names, sizes, and boundaries" (Coulton et al, 2011). Hand-drawings on paper maps of neighbourhoods from the Making Connections study have also been used to investigate the relationship between "mental maps" and within-city migratory patterns (English, 2007). Our study makes use of digitized HDRN maps from the Making Connection study to explore to what degree study participants draw their HDRN as a circle and to recommend an optimal radius for measuring attributes of the residential neighbourhood with circular spatial buffers.

Studies over a wide variety of disciplines have used Geographic Information System (GIS) software to estimate circular spatial buffers to study: air pollutants (Ross et al, 2013); political elections (Linke, 2013); neighbourhood walkability (Duncan et al, 2013); mammalian behavior in suburban yards (Kays \& Parsons, 2014); adolescent sexual behaviors (Bersamin, Todd, \& Remer, 2011); gas shale exploration effects on housing prices (Gopalakrishnan \& Klaiber, 2014); body mass index (Lear et al, 2011); remote sensing and malaria (Benali et al., 2014); vehicle traffic patterns (Pulugartha \& Kusam, 2012); toxic release effects (Conley, 2011); adverse birth outcomes (Meng et al., 2013); physical activity (Colabianchi et al, 2014); and many others. A complete review of the literature using circular spatial buffers would include thousands of publications extending back decades.

Our study hopes to contribute towards standardizing the use of circular special buffers when developing measures of the residential environment. Our conceptual framework is founded on the testable hypothesis that most humans possess a cognitive representation of what they perceive as their residential neighbourhood. We presume the cognitive representation is accompanied by a relatively stable delineation in the geographical boundaries of the meaningful physical space. Although formal testing of this hypothesis is plausible through empirical methods, it remains untested until now. The, frequently implicit, theoretical axiom that most humans possess a cognitive representation of the geographical properties of meaningful physical spaces is the primary motivator in research attempting to measure aspects of the neighbourhood as experienced by residents. At its core, the hypothesis posits humans have the ability and habit of assigning relatively static geographical characteristics to meaningful physical spaces.

Our study investigated to what extent resident drawn shapes of their neighbourhoods were circular. The hand-drawn shapes were presumed to reflect an individual's cognitive map on the geographical properties of their residential neighbourhood. Thus, cognitive maps in this paper refer to hand-drawn maps 
from "spatial knowledge acquired from maps and navigation" (Thorndyke \& Hayes-Roth, 1982) and not a model of reasoning (Eden, 1992). In order to determine an optimum radius size when using circular spatial buffers to measure attributes of the residential neighbourhood, we quantified the degree to which adults drew their residential neighbourhood as a circle. We supplemented the main goals by investigating how level of circularity varies as a function of demographic factors.

\section{Methods}

Data

We used HDRN maps and data from the Making Connections study. Makings Connections is a community change initiative sponsored by the Anne E. Casey Foundation (AECF). The Making Connections target areas were chosen through a deliberative process involving the Foundation and local representatives to include distressed neighbourhoods with concentrations of poverty and disproportionately representative of immigrants or racial and ethnic minority groups. According to documentations by data creators of the Making Connections study, extensive efforts were made to give each household within the target areas area an equal chance of selection. Survey data was collected jointly by the National Opinion Research Corporation (NORC) at the University of Chicago and the Urban Institute. Participants gave consent under the supervision of NORC. The current analysis only uses de-identified Making Connections data. As required by NORC and AECF, our analysis of secondary data received approval by the Institutional Review Board at the University of Pittsburgh (REN1412008, PRO13100013) in Pennsylvania.

\section{Sample}

The sample comes from individuals who completed Wave 1 of the Making Connections survey between 2002 and 2003. A total of 6,674 households were interviewed from multiple areas within the following cities: Des Moines, IA $(\mathrm{n}=779)$; Indianapolis, IN $(\mathrm{n}=786)$; Denver, CO $(\mathrm{n}=785)$; Seattle, WA $(\mathrm{n}=791)$; Milwaukee, WI $(\mathrm{n}=701)$; Oakland, CA $(\mathrm{n}=703)$; Hartford, CT $(\mathrm{n}=697)$; Providence, RI $(n=697)$; and Louisville, KY $(n=735)$. Eighty-three percent $(83 \%)$ of study participants drew lines representing their residential neighbourhood on paper maps. Because we were interested in understanding the predictors of level of circularity, we omitted observations without information on sex, age, race, ethnicity, educational attainment, and number of months living in the neighbourhood. Thus, our analysis included a total of 4,742 observations. Because of our selection protocol and the fact that data were collected in nonrandomly selected metropolitan areas in the US, we advise caution in generalizing our findings beyond the sample under analysis.

Drawing Maps

Trained interviewers presented coloured paper maps to respondents that covered an area larger than their residential area. The printed paper map 
contained key landmarks and selected street names to orient the study participant. After the interviewer had identified the respondent's home on the paper map, they ask the study participant to pencil-draw the boundaries of what they perceived as their neighbourhood and were read the following statement:

By neighbourhood, I mean the area around where you live and around your house. It may include places you shop, religious or public institutions, or a local business district. It is the general area around your house where you might perform routine tasks, such as shopping, going to the park, or visiting with neighbors. Please take a look at this map of the area. Study it for a moment and use this pencil to draw the boundaries of what you consider your neighbourhood.

Note that although the term "residential neighbourhood" does not appear in the question, the explanation of what the interviewer meant by neighbourhood includes the idea that the construct referred to the physical space 'around the house.'

Admittedly, the statement defining neighbourhood for map-drawers also referenced 'general areas around the house' where they may 'perform routine tasks' such as shopping. For some study participants, such as those who shop for groceries and amenities well beyond their place of residence, the definition of the neighbourhood may be said to be "double-barreled"- - we return to potential implications of this instruction in the following section. When asking study participants to draw their neighbourhood map, interviewers were allowed to prompt respondents to pencil-mark a closed polygon representing the geographical boundaries of what the respondent perceived as their residential neighbourhood. In our study, we assume that hand-drawings on paper maps approximate the geographical boundaries as individual references when thinking of their residential neighbourhood.

\section{Digitizing maps}

In order to investigate the geometrical properties of hand-drawn maps, paper drawings had to be digitized. Paper maps with analog sketches were originally stored at field centers charged with collecting baseline data. Some paper maps faced special challenges, such as incomplete sketches; incomplete polygons; and unclear neighbourhood boundaries. Using sensitive data quality control measures, approximately $10 \%$ (from 6,224) of the maps were flagged for some irregularity by field centers conducting interviews. As per internal technical documentation, paper maps containing abnormalities received customized attention by technicians at NORC. These analog sketches with abnormalities were referred to as exceptions.

Most exceptions could be generally categorized as having: multiple areas sketched; lines going off the map; unclosed polygons; or pencil lines that circled street names or landmarks instead of an area per-se. Most exceptions with lines going off map were handled by drawing a borderline to the nearest natural boundary (e.g., river, highway). Most analog sketches with unclosed polygons were interpreted with ease as only small line segments were missing. Some incomplete polygons, like sketches, only containing scattered elements, required more qualitative inference on the part of the NORC supervised technician. 
A polygon was qualitatively interpolated by NORC supervised technicians to enclose indicated points in maps where street names and/or landmarks were circled.

Sketches drawn on color paper maps were made machine-readable (i.e., digitized) by converting them to electronically archived equivalent elements (points, lines, and polygons) of planar geometry (Cowen, 1988). More technically, analog sketches (i.e., paper drawings) were converted to electronic files by using CalComp Drawing Board III digitizing tables (12" by 18"), in Windows 2000 environment.

The two CalComp digitizers were equipped with four-button pucks to facilitate tracing on paper maps. The software component, MapInfo ${ }^{\circledR}$ Professional 7.0, communicated with the hardware component (i.e., CalComp digitizers), in MapInfo ${ }^{\circledR}$ format by using a systematized protocol that insured data quality in six stages: (1) examination of maps before digitizing; (2) hardware and software setup; (3) map registration; (4) digitizing process; (5) postprocessing and exception handling; and (6) validation.

"Registration" refers to the process used for relating the location of a paper map to the geographic coordinates of longitude and latitude. After firmly affixing paper map to CalComp tablet by using a transparent drop sheet to prevent movement or wrinkles, known the longitude and latitude points were set as controls. In order for MapInfo® Professional 7.0 to complete the affine transformations, necessary for deriving latitude and longitude for the entire plane, each paper map received four control points. Trained technicians digitized paper maps to create native MapInfo® mapable files. The "polygon tool" in the "cosmetic layer" was used to identify control points whose error value had to be $<0.015$ inches. When the CalComp puck was in control, the technician digitized the paper map by tracing the lines sketch by study participants. Technicians were instructed to add sufficient points on curved objects so as to avoid jagged edges.

As per technical documentation at NORC, exceptional procedures were undertaken to ensure the quality of work in digitized maps. After visually comparing paper and digital maps, maps with errors were re-digitized. A random sample of maps was digitized twice for comparison. Given the thick felttipped CalComp marker, the scale of the paper map, and free-hand nature of sketching, some displacement could occur for up to 100 feet in some cases. The displacement of some digitized maps is inconsequential to our analysis but may play a major role in other studies looking at polygon centroids. Ultimately, digitized maps were stored in MapInfo files.

The lead author of this paper converted existing MapInfo® files to ArcGIS® format (i.e., shapefiles) by using QGIS 2.4 software. Maps were stored using the North American Datum of 1983 (NAD83) - the geodetic network most commonly used to define North America. Digitized polygons were projected in ArcMap® 10.2 using USA Contiguous Albers Equal Area Conic USGS-where scale and shape are not preserved, and distortion between standard parallels is kept to a minimum. The Albers Equal Area Conic projection helps maintain maximum area accuracy across the contiguous US. All the metropolitan areas under investigation are within the US mainland. 


\section{Level of circularity}

The level of circularity was derived by using minimum bounded geometry procedures in ArcMap ${ }^{\circledR}$ 10.2. As mentioned earlier, the level of circularity refers to what degree a study participant drew his or her HDRN as a circle. Using ArcMap ${ }^{\circledR}$ 10.2, we measured the Minimum Bounded Circle (MBC) for each HDRN. To compute MBC, we took the two points most distant from oneanother within the set of points making up the digitized HDRN polygon. We then used the straight line between these two most distant points to calculate a diameter and infer the smallest possible circle capable of enclosing the HDRN map. More technically, MBC is determined by using the largest within-HDRNpolygon diameter. The MBC represents the smallest circle enclosing the HDRN polygon. Figure 1 presents elements used in computing the MBC. After we had determined the area within the HDRN and MBC using square miles (mi2), we computed level of circularity as follows:

$$
\text { Level of Circularity }=\left(\frac{\text { HDRN area in } \mathrm{mi}^{2}}{\text { MBC area in } \mathrm{mi}^{2}}\right) \times 100
$$

The level of circularity is the percent of the HDRN within the MBC. For example, if a hypothetical HDRN has an area of $0.33 \mathrm{mi}^{2}$ and an MBC area of $0.44 \mathrm{mi}^{2}$, then: $[(0.33 \div 0.44) \times 100]=75 \%$, indicating $75 \%$ of the HDRN is contained within the MBC. Figure 2 displays three hypothetical scenarios to show how the level of circularity can vary from high, to moderate, and low. As ours is the first publication ever to measure level of circularity in digitized HDRN maps, we do not have any guidance on what should be considered a "low" level of circularity. Labels such as high, moderate and low are used to ease the discussion.

\section{Optimum radius}

After determining level of circularity, we determined the optimal radius size of residential neighbourhood in our study with the following equation:

$$
\text { Optimum Radius }=\left[\text { MBC diameter } \times\left(\frac{\text { Level of Circularity }}{100}\right)\right] \div 2
$$

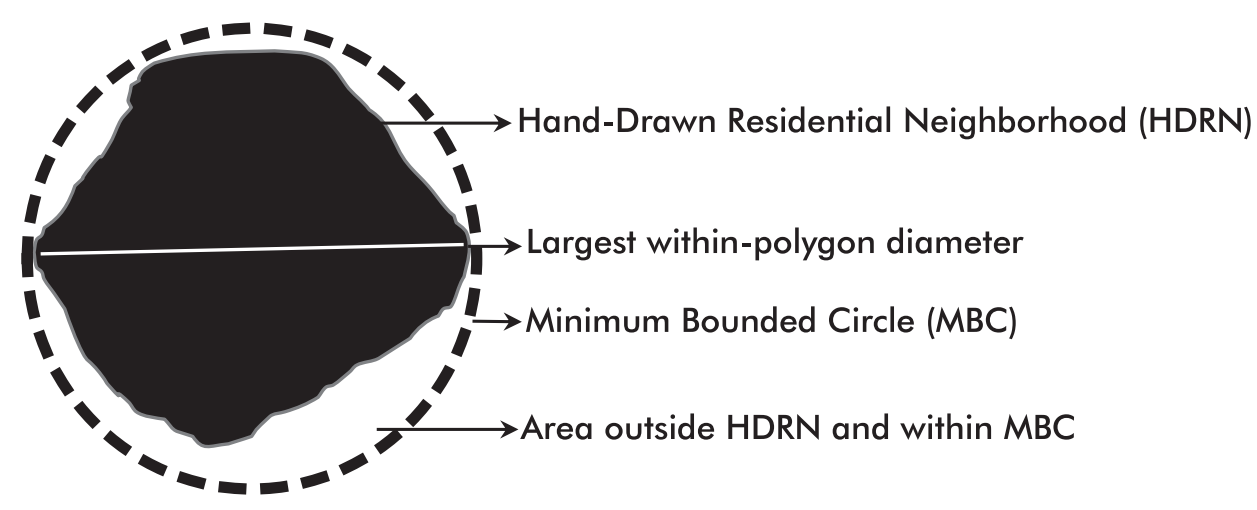

Figure 1. Areas used for computing level of circularity 

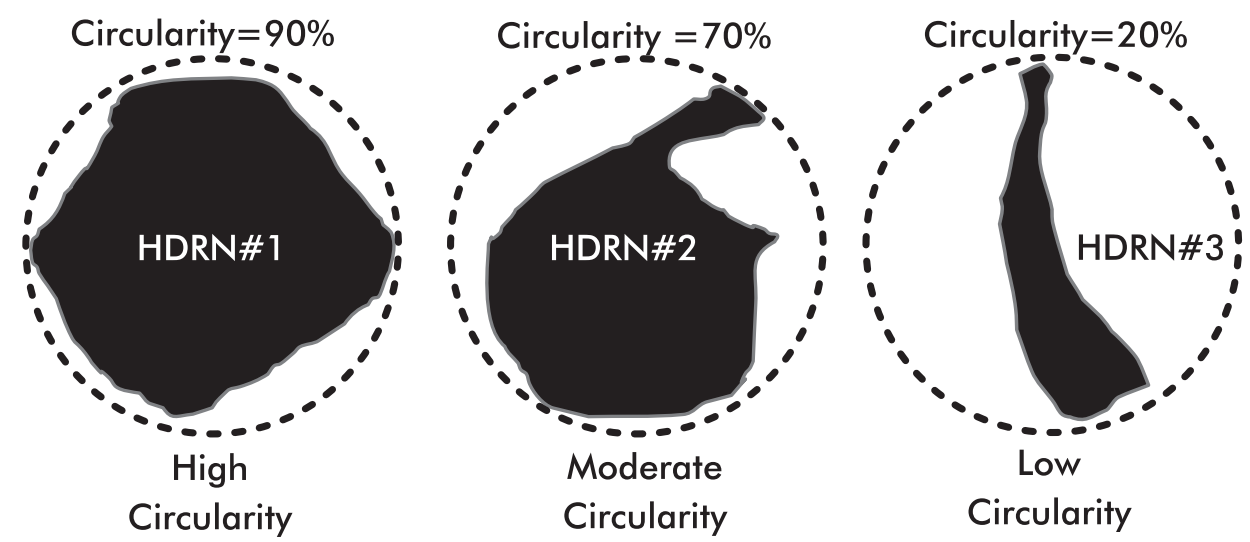

Figure 2. Hypothetical examples in levels of circularity

The optimum radius for each study participant is computed by deriving the product of the MBC diameter with the quotient of the level of circularity and 100 and dividing the result by two. MBC diameter is adjusted for level of circularity to account for how circular the individual drew the geographical boundaries of his or her residential neighbourhood. It is divided by two to obtain the radius of the diameter.

For example, assume a hypothetical scenario: $\mathrm{MBC}$ has a diameter of 0.66 miles, and a level of circularity is 75 , then: $[0.66 \times(75 \div 100)] \div 2 \approx 0.25$. In this scenario, it may be advisable to measure characteristics of the residential neighbourhood for this individual by using a circular spatial buffer that has a 0.25 mile radius. Optimum radius size is the main measure of interest and is computed for each study participant in order to derive the most recommendable radius size when measuring attributes of the residential environment via circular spatial buffers.

\section{Covariates}

In addition to providing researchers with the optimum radius size when measuring aspects of the residential environment, we delineate level of circularity in HDRN by basic demographic factors and model level of circularity while including the following variables: sex; age; race; ethnicity; educational attainment; and months living in the residential neighbourhood. We decided to focus on an individual's demographic characteristics to provide researchers a sense of the appropriateness of circular buffers for various groups.

Age, ranging from 18 to 75, is measured continuously. Age is included as tasks in activities of daily living may differ over the life-course. We account for sex, with females as the reference category, because labor participation and household responsibilities (which differs by sex) may play a role in how adults navigate and perceive the physical environment. Educational attainment is accounted for as it is a proxy measure of access to socioeconomic resources. Educational attainment was assessed in Making Connection by using an adjectival scale with the following 9 categories: $\leq 8^{\text {th }}$ grade or less $(12 \%)$; $9^{\text {th }}$ grade but no high school (HS) diploma; General Education Development (GED); HS diploma; trade of vocational school; 1 to 3 years of college; and $\geq$ graduated four-year college. We separate individuals 
into three groups: $\leq \mathrm{HS}$; some college; and $\geq$ graduated four-year college (reference group).

We account for months living in the residential neighbourhood as it may play a role in how individuals are able to specify the geographical boundaries of meaningful places around the residential area. A number of years and months living in the neighbourhood was assessed in Making Connection by asking: How long have you lived in this neighbourhood? Time in the neighbourhood is measured continuously as a total number of months. Because the size of the digitized polygon varies widely between Making Connections participants, we control for the size of the drawn area by including the diameter (in miles) of the MBC.

\section{Statistical analysis}

We regressed on the level of concentricity by using female, age, race-ethnicity, educational attainment, months in a residential neighbourhood, and diameter in minimum bounded circle. While Models 1 through 4 include all 4,742 observations under analysis, "Model-5" omits observations $(n=455)$ where map drawings required specialized attention. This is done as a way of testing potential bias produced from 'exception review' protocols (e.g., tracing a boundary that encircles multiple polygons into one).

\section{Results}

Distribution in level of circularity by basic demographics

Because our dependent variable is level of circularity, we provide its distribution stratified by basic demographic factors of sex, race, and ethnicity. From a qualitative review of the visual information in Figure 3, it appears level of circularity in HDRN maps is low to moderate $(\leq 70.4)$ for about $70 \%$ of people and

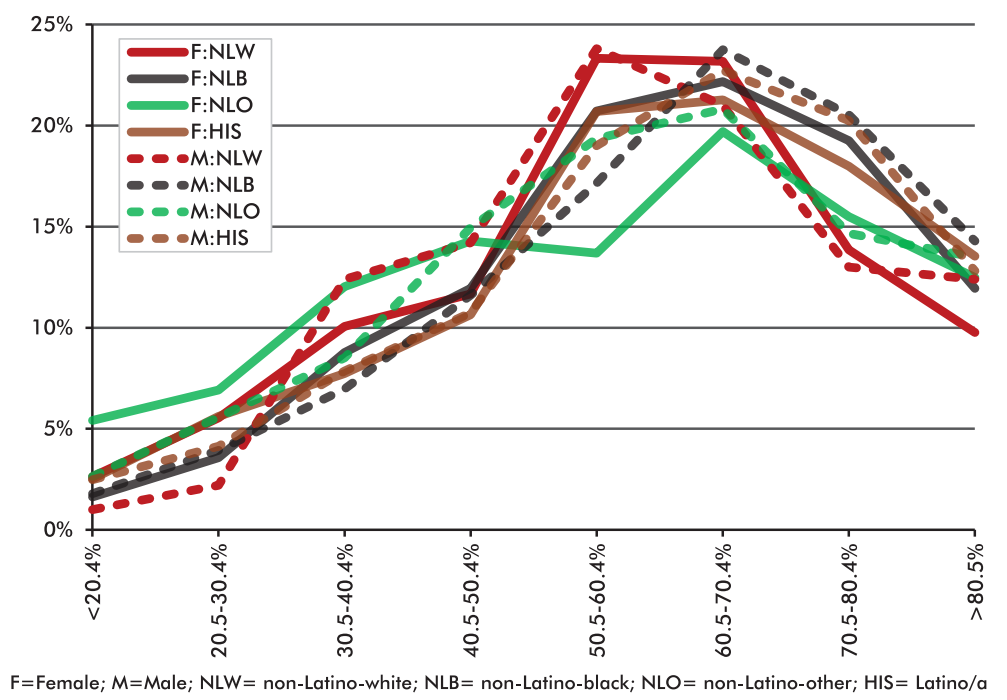

Figure 3. Distribution of "level of circularity" from hand-drawn maps of participant-defined residential neighborhood 


\section{Siordia E Coulton}

has a similar distribution across all groups. The stability of the pattern of the distribution of level of circularity provides some indication that similar factors may be helping influence to what degree individuals draw the geographical boundaries of their residential neighbourhood as a circle.

The fact that level of circularity in HDRN maps is relatively stable between sexrace-groups may give some credence that adults from multiple metropolitan areas in the US and in the Making Connections sample possess a cognitive representation of the residential neighbourhood that is influenced by potentially measurable factors. That is, the stability in the distribution of level of circularity between sex-race groups may provide some anecdotal evidence that humans have the ability and habit of assigning geographical characteristics to meaningful spaces in both a systematic and measurable way.

\section{Level of circularity by site}

We provide descriptive statistics by site as the geographical, and other properties vary between the cities in Making Connections. Tables 1 and 2 provide descriptive statistics for all study variables by site-ordered by decreasing the level of circularity. Average levels of circularity, from highest to lowest were as follows: Hartford at 66; Louisville, Oakland, and Providence at 64; Milwaukee at 63; Seattle at 62; Seattle at 62; Indianapolis and Des Moines at 56; and Denver with the lowest level of circularity at 38 . These numbers indicate residential neighbourhoods were on average not drawn as a circle. For most study participants, HDRN polygons only covered about half the area of the MBC. The numbers in Table 1 suggest most individuals drew non-circular geographical boundaries to depict their residential neighbourhood-especially in Denver.

\section{Optimum radius}

Table 1 shows the optimum radius, and listed from lowest to largest are as follows: Hartford at 0.17; Denver at 0.18; Providence at 0.21; Oakland at 0.24; Milwaukee at 0.27; Indianapolis at 0.32; Des Moines at 0.33; Seattle at 0.37; and Louisville at 0.42 . These 9 optimum radii range from 0.17 to 0.42 . The nine optimum radii have a medium of 0.27 and average to 0.28 . In answer to our main research question, when using circular spatial buffers to measure environmental characteristics of the residential neighbourhood, the best radius size is 0.25 miles. We recommend a $1 / 4$ mile radius for measuring attributes of the residential neighbourhood with circular spatial buffers as it is already widely used and more conceptually cohesive than a 0.28 -mile radius.

From Table 1 we see that in most sites, females made up $60 \%$ to $70 \%$ of the sample. Louisville had the largest percent of Non-Latino-Blacks (79\%). The Des Moines site had the largest percent of Non-Latino-White (53\%), the Oakland site the largest group of Non-Latino-Others (39\%), and Providence the largest group of Latinos/Hispanics (38\%). As indicated by the average number of months residing in the neighbourhood, most individuals on average resided in their residential area for 5 or more years. In most study sites, the majority of participants reported having a High School educational attainment or below. 


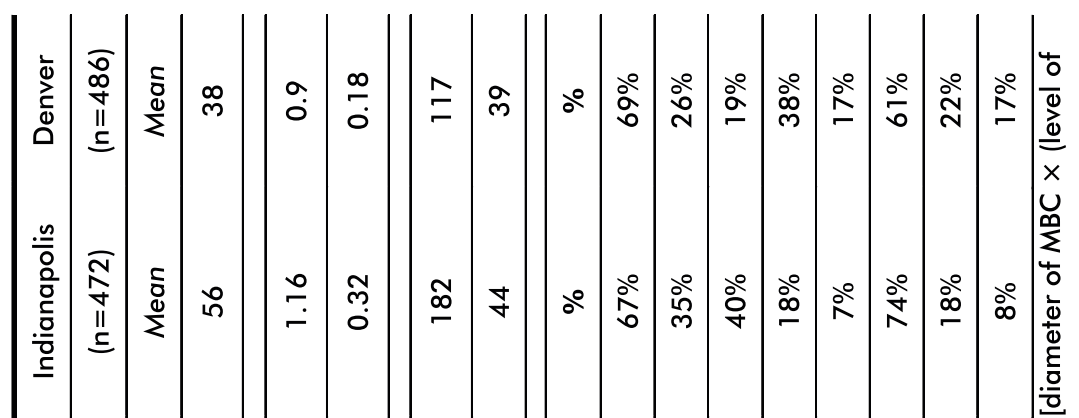

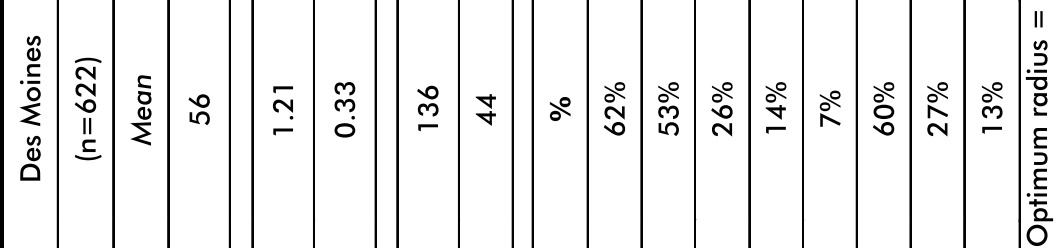

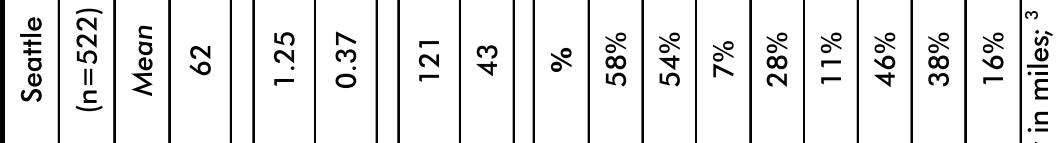

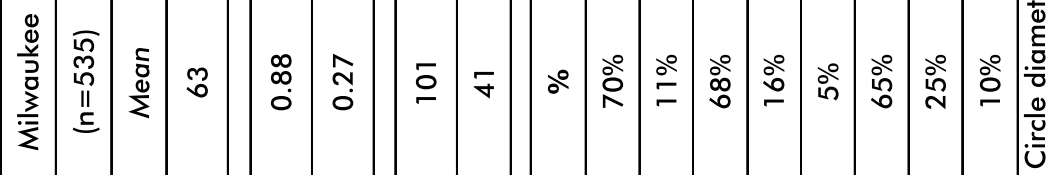

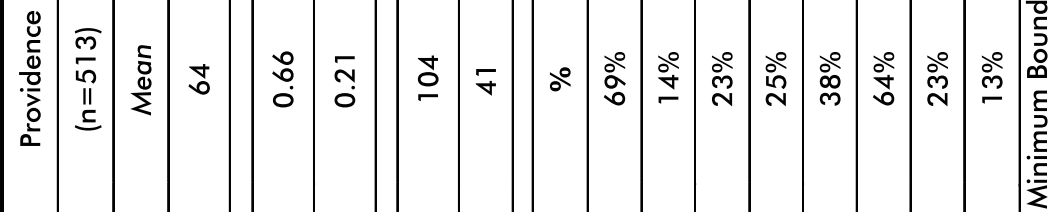

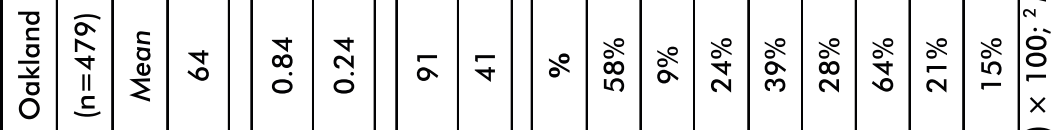

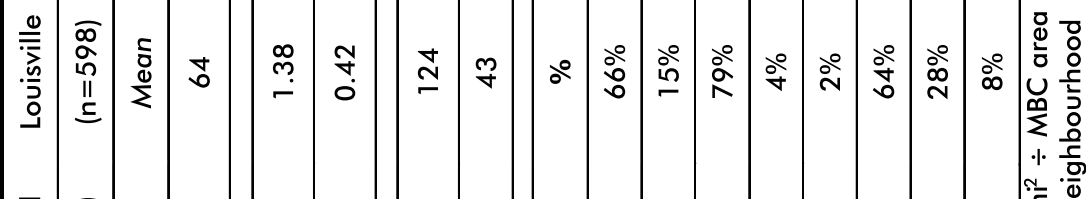

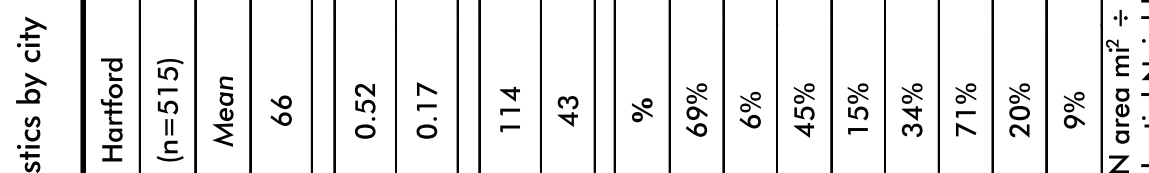

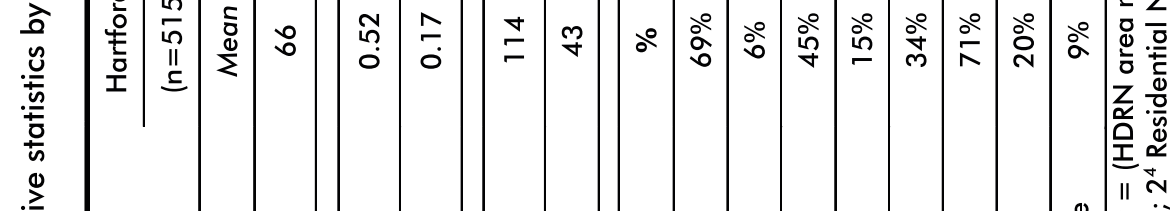

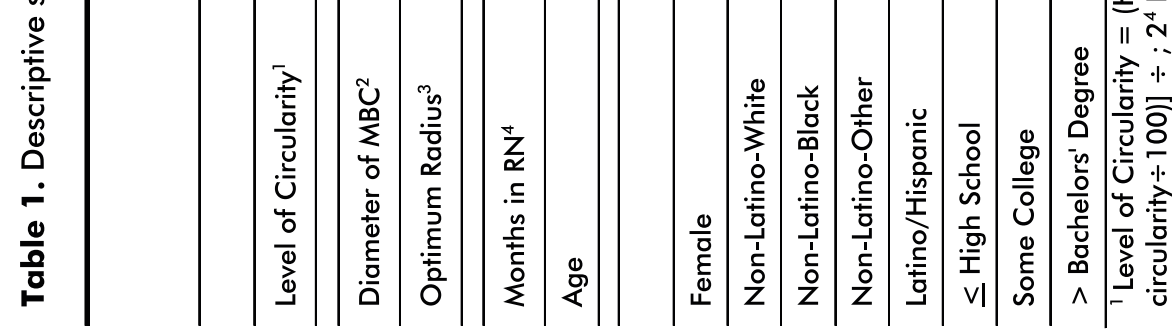


Regressions predicting level of circularity

Table 2 provides the results of our exploratory regression analysis. The objective of these regressions was to explore how basic demographic factors are associated with the level of circularity in HDRN maps. Only statistically significant results at the 0.05 level or below are discussed. As can be seen from all the models, females seem to draw polygons of a residential neighbourhood that are less $(\beta=-1.63)$ circular than males. This may be interpreted as indicating that females draw less circular residential neighbourhoods than males. From Model-4, which includes drawings with edited anomalies, we find that when compared to Non-Latino-Whites: NonLatino-Blacks drew more $(\beta=2.61)$ circular polygons and Non-Latino-Others drew less $(\beta=-1.79)$ circular polygons to represent what they perceive as their residential neighbourhood. This may indicate Non-Latino-Blacks perceive their residential neighbourhood less circular than Non-Latinos-Whites.

We also find residential stability is associated with drawing less circular neighbourhoods. From the covariate accounting for the individuals MBC diameter, we see evidence that individuals who drew larger polygons also provided less circular pencil drawings of their residential neighbourhood. In general, models showed that age is not a statistically significant predictor of neighbourhood circularity. Education (i.e. having some college compared to a bachelor' s degree) is shown to have a significant effect on circularity only after controlling for residential stability and MBC (Model 4). As evident from results in Model-5, the findings remain the same (similar to Model-4) even after drawings with anomalies are excluded from the analysis.

\section{Discussion}

The findings provide some practical information to guide researchers who are considering using spatial buffers to represent neighbourhoods in their studies. However, it is difficult to determine the causal mechanisms between our predictors and their association with level of circularity. It may be speculated that the more individuals interact with their residential environments; the less likely they are to draw a circular polygon. In other words, a tendency towards drawing a more circular polygon may be a sign of low familiarity with the residential environment. After spending more time traversing the areas around their homes, residents may come to include or exclude specific portions in their own conception of the neighbourhood. Moreover, individuals with less exposure or a smaller zone of comfort in the place may be less discerning when asked to map of their neighbourhood. The observed differences in circularity by gender, race and ethnicity, education and longevity of residency may be reflective of these varying experiences.

There are several limitations of this study that must be considered. First, the sample is not representative of the 9 cities, but only of those target areas chosen for the Making Connections initiative. As these were required to be low-income and racially and ethnically diverse areas, the findings cannot be generalized to other types of areas. Second, the instructions provided to the respondents for drawing their maps may have inadvertently introduced some measurement error.

As noted above, approximately 10 percent of the maps had some anomalies. Making Connections interviewers defined neighbourhood for map-drawers in such a way that 'general areas around the house where routine tasks such as shopping' were included along with the area around the house. 
Table 2. Results from multivariate linear regressions predicting "level of circularity"

\begin{tabular}{|c|c|c|c|c|c|c|c|c|c|c|}
\hline & \multicolumn{2}{|c|}{ Model-1 } & \multicolumn{2}{|c|}{ Model-2 } & \multicolumn{2}{|c|}{ Model-3 } & \multicolumn{2}{|c|}{ Model-4 } & \multicolumn{2}{|c|}{ Model-5 ${ }^{1}$} \\
\hline Female & -1.33 & $*$ & -1.53 & $* *$ & -1.57 & $* *$ & -1.63 & $* *$ & -1.50 & $* *$ \\
\hline Age & 0.003 & & 0.004 & & 0.004 & & 0.02 & & 0.02 & \\
\hline Non-Latino-White & & & Ref & & Ref & & Ref & & Ref & \\
\hline Non-Latino-Black & & & 2.83 & $* * *$ & 2.67 & $* * *$ & 2.61 & $* * *$ & 2.61 & $* * *$ \\
\hline Non-Latino-Other & & & -0.93 & & -1.04 & & -1.79 & $* *$ & -1.68 & $*$ \\
\hline Latino/Hispanic & & & 2.32 & $* *$ & 2.17 & $* *$ & 0.87 & & 1.07 & \\
\hline$\leq$ High School & & & & & 1.19 & & 0.68 & & 1.49 & \\
\hline Some College & & & & & 1.61 & & 1.68 & * & 2.27 & $* *$ \\
\hline$\geq$ Bachelors' Degree & & & & & Ref & & Ref & & Ref & \\
\hline Months living in $\mathrm{RN}^{2}$ & & & & & & & -0.01 & $* *$ & -0.01 & $*$ \\
\hline Diameter of $\mathrm{MBC}^{3}$ & & & & & & & -0.01 & $* * *$ & -0.01 & $* * *$ \\
\hline Intercept & 60.1 & $* *$ & 58.9 & $* * *$ & 57.9 & $* * *$ & 62.2 & $* * *$ & 61.8 & $* * *$ \\
\hline $\begin{array}{l}{ }^{*} \leq 0.05 \text {; }^{* *} \leq 0.01 ;{ }^{* * *} \\
\text { ' Unlike models } 1 \text { throu } \\
\text { complete analysis, Mo } \\
\text { where hand-drawn pol } \\
{ }^{2} \mathrm{RN}=\text { Residential neigh } \\
{ }^{3} \mathrm{MBC}=\text { Minimum boun }\end{array}$ & $\begin{array}{l}0.001 \\
\text { h } 4 \text { whic } \\
\text { del-5 only } \\
\text { gons we } \\
\text { orhood } \\
\text { ed circle }\end{array}$ & $\begin{array}{l}\mathrm{h} \text { in } \\
\text { inc } \\
\text { re N }\end{array}$ & $\begin{array}{l}\text { de all } \\
\text { des the } \\
\text { T flagge }\end{array}$ & 742 & $\begin{array}{l}\text { servati } \\
0 \% \text { ) ob } \\
\text { nor dis }\end{array}$ & $\begin{array}{l}\text { ns in } \\
\text { serva } \\
\text { repa }\end{array}$ & $\begin{array}{l}\text { he } \\
\text { ons } \\
\text { cies. }\end{array}$ & & & \\
\hline
\end{tabular}

As noted above, approximately 10 percent of the maps had some anomalies. Making Connections interviewers defined neighbourhood for map-drawers in such a way that 'general areas around the house where routine tasks such as shopping' were included along with the area around the house.

Although the definition of neighbourhood may have been intended to encourage study participants to envision a single spatial entity (i.e., one polygon), referencing multiple examples may have created more heterogeneity in what study participants understood. For example, for individuals whose daily activities cover a large geographical space (e.g., $\geq 5$ miles), areas, where routine tasks are conducted, may be well beyond the place of residence. For individuals with large lived-spaces, the inclusion of multiple examples in the definition of the neighbourhood may have made the instructions double-barreled: the definition included multiple geographical areas while only intending to conjure the geographical boundaries of one location. This may help explain why multiple polygons were drawn on some paper maps. It may also illuminate why some Making Connections study participants drew pencil lines going off the map provided by interviewers - as other meaningful spaces may have been well beyond the area of residence. According to technical documentation by NORC, paper map exceptions with multiple sketches were digitized by considering the largest drawn 
areas (outer area of all sketches). The qualitatively derived procedure assumed respondents intended to define multiple areas as a contiguous neighbourhood.

\section{Conclusions}

We have provided empirical evidence from the quantitative analysis that when using circular spatial buffers to measure environmental characteristics of the residential neighbourhood as perceived by residents, the optimal radius size is 0.25 miles. We also found evidence that sex, race, ethnicity, education, size of HDRN and number of months living in neighbourhood help explain the variance between individuals in the level of circularity in HDRN. As potential support for the idea that humans have the ability and habit of assigning relatively static geographical characteristics to meaningful physical spaces we found most individuals-across multiple metropolitan areas and demographic backgrounds-drew the boundaries of their residential neighbourhood with a moderate level of circularity.

As of the publication of this research, no empirically informed techniques are available to delineate optimal procedures for systematically fixing/editing pencil map-drawing anomalies. The Making Connections study is a researcher-trailblazer capable of helping form the basis for how to advance the empirical quantification in the geographical properties of meaning places. Notwithstanding limitations, ours is the first study to empirically determine the recommendable radius size when measuring environmental attributes of the residential environment using circular spatial buffers. Our results may help promote the establishment of measurement protocols that increase inter-study comparability. By making use of unique data from the Making Connections study, we have demonstrated basic geographical properties of HDRN maps. Further research along these lines in additional places and with more varied samples can move neighbourhood research towards better understanding the complex mechanism through which humans assign geographical boundaries to meaningful places.

\section{References}

Benali, A, Nunes, JP, Freitas, FB, Sousa, CA, Novo, MT, Lourenço, PM, Lima, JC, Seixas, J \& Almeida, APG 2014, 'Satellite-derived estimation of environmental suitability for malaria vector development in Portugal', Remote Sensing of Environment, vol. 145, pp. 116-130.

Bersamin, M, Todd, M \& Remer, L 2011, 'Does distance matter? Access to family planning clinics and adolescent sexual behaviors', Maternal and Child Health Journal, vol. 15, no. 5, pp. 652-659.

Callaghan, WM 2014, 'Geographic variation of reproductive health indicators and outcomes in the United States: place matters', American Journal of Obstetrics and Gynecology, vol. 211, no. 3, pp. 278-284.

Chaix, B 2009, 'Geographic life environments and coronary heart disease: a literature review, theoretical contributions, methodological updates, and a research agenda', Annual Review of Public Health, vol. 30, pp. 81-105.

Colabianchi, N, Coulton CJ, Hibbert JD, McClure, SM, Ievers-Landis, CE \& Davis, EM 2014, 'Adolescent self-defined neighbourhoods and activity spaces: spatial overlap and relations to physical activity and obesity', Health \& Place, vol. 24, pp. 22-29.

Conley, JF 2011, 'Estimation of exposure to toxic releases using spatial interaction 
modeling', International Journal of Health Geographies, vol. 10, no. 20.

Coulton, C, Chan T, Mikelbank K 2011, 'Finding place in community change initiatives: Using GIS to uncover resident perceptions of their neighbourhoods', Journal of Community Practice, vol. 19, pp. 10-28.

Coulton, CJ, Cook T, Irwin M 2004, Aggregation issues in neighbourhood research: A comparison of several levels of census geography and resident defined neighbourhoods, Case Western Reserve University, Cleveland.

Coulton, CJ, Korbin J, Chan T, Su M 2001, 'Mapping resident perceptions of neighbourhood boundaries', American Journal of Community Psychology, vol. 29, no. 2, pp. $371-383$.

Cowen, DJ 1988, 'GIS versus CAD versus DBMS--what are the differences' Photogrammetric Engineering Eं Remote Sensing, vol. 54, pp. 1551-1555.

Dorling, D 2010, Injustice: Why social inequality persists, Policy Press, Bristol.

Duncan, DT, Aldstadt, J, Whalen, J \& Melly SJ 2013, 'Validation of walk scores and transit scores for estimating neighbourhood walkability and transit availability: a small-area analysis', GeoJournal, vol. 78, pp. 407-416.

Duncan, OD 1957, 'The measurement of population distribution', Population Studies, vol. 11, pp. 27-45.

Eden, C 1992, 'On the nature of cognitive maps', Journal of Management Studies, vol. 29, no. 3, pp. 261-265.

Ellen, IG, Mijanovich, T \& Dillman, KN 2001, 'Neighbourhood effects on health: exploring the links and assessing the evidence', Journal of Urban Affairs, vol. 23, no. 34, pp. 391-408.

English, E 2007, Using mental maps to study neighbourhood movement in the inner-city: Formal vs. informal definitions, Presentation at the Association of American Geographers Annual Meeting.

Gaskin, DJ, Thorpe Jr, RJ, McGinty, EE, Bower, K, Rohde, C, Young, JH, LaVeist, TA \& Dubay, L 2014, 'Disparities in diabetes: the nexus of race, poverty, and place', American Journal of Public Health, vol. 104, no. 11, pp. 2147-2155.

Gavin, VR, Seeholzer, EL, Leon, JB, Chappelle, SB \& Sehgal, AR 2015, 'If We Build It, We Will Come: A Model for Community-Led Change to Transform Neighbourhood Conditions to Support Healthy Eating and Active Living', American Journal of Public Health, vol. 105 , no. 6 , pp. 1072-1077.

Ghosh, SK \& Goswami PP 2013, 'Unsolved problems in visibility graphs of points, segments, and polygons', ACM Computing Surveys (CSUR), vol. 46, no. 22.

Gopalakrishna, S \& Klaiber, HA 2014, 'Is the shale energy boom a bust for nearby residents? Evidence form housing values in Pennsylvania', American Journal of Agricultural Economics, vol. 96, no. 43-66.

Gupta, A \& Ferguson, J 1992, 'Beyond "culture": space, identity, and the politics of difference', Cultural Anthropology, vol. 7, no. 6-23.

Houston, D 2014, 'Implications of the modifiable areal unit problem for assessing built environment correlated of moderate and vigorous physical activity', Applied Geography, vol. 50; pp. 40-47.

Huh, J, Paul Thing, J, Abramova, ZS, Sami, M \& Beth Unger, J 2014, 'Place Matters in Perceived Tobacco Exposure among Korean American Young Adults: Mixed Methods Approach', Substance Use Ė Misuse, vol. 49, no. 8, pp. 1054-1063.

James, P, Berrigan, D, Hart, JE, Hip, JA, Hoehner, CM, Kerr, J, Major, JM, Oka, M \& Laden, F 2014, 'Effects of buffer size and shape on associations between the built environment and energy balance', Health छ Place, vol. 27, pp. 162-170.

Kays, R \& Parsons, AW 2014, 'Mammals in and around suburban yards, and the attraction of chicken coops', Urban Ecosystems, vol. 17, pp. 691-705.

Kirkpatrick, D 1983, 'Optimal search in planar subdivisions', SIAM Journal on Computing, vol. 12 , no. 1 , pp. 28-35.

Kwan, MP 2012a, 'The uncertain geographic context problem', Annals of the Association of 


\section{Siordia E Coulton}

American Geographers, vol. 102, pp. 958-968.

Kwan, MP 2012b, 'How GIS can help address the uncertain geographic context problem in social science research', Annals of GIS, vol. 18, no. 4, pp. 245-255.

Kytta, AM, Broberg, AK \& Kahila, MH 2012, 'Urban environment and children's active lifestyle: SoftGIS revealing children's behavioral patterns and meaningful palaces', American Journal of Health Promotion, vol. 26, no. 5, pp. e137-e148.

Leal, C, Bean, K, Thomas, F \& Basile, C 2011, 'Are associations between neighbourhood socioeconomic characteristics and body mass index or waist circumference based on model extrapolations?', Epidemiology, vol. 22, no.5, pp. 694-703.

Lefebvre, H 1991, The production of space, Blackwell, Oxford.

Lewin, K 1951, 'Formalization and progress in psychology' in D Cartwright (ed), Field Theory in Social Sciences, New York, Harper.

Linke, AM 2013, 'The aftermath of an election crisis: Kenyan attitudes and the influence of individual-level and locality violence', Political Geography, vol. 37, pp. 5-17.

Matthews, SA \& Yang, TC 2013, 'Spatial polygamy and contextual exposures (SPACEs) promoting activity space approaches in research on place and health', American Behavioral Scientist, vol. 57, no.8, pp. 1057-1081.

Meng, G, Hall, GB, Thompson, ME \& Seliske, P 2013, 'Spatial and environmental impacts on adverse birth outcomes in Ontario', The Canadian Geographer/Le Géographe canadien, vol. 57 , no. 2, pp. 154-172.

Newburger, H, Birch, EL \& Wachter, SM 2011, Neighbourhood and life chances: how place matters in modern America, University of Pennsylvania Press.

Nicholson, V 1969, 'Mapping cylinder neighbourhoods', Transactions of the American Mathematical Society, vol. 143, pp. 259-268.

Nurmi, P \& Bhattacharya, S 2008, 'Identifying meaningful places: the non-parametric way', Pervasive Computing/Springer Berling Heidelberg, vol. 5013, pp. 111-127.

Openshaw, S 1984, The modifiable areal unit problem, Geo Books, Norwich, England.

Petterson, R \& Zillinger, M 2011, 'Time and space in event behavior: tacking visitors by GPS', Tourism Geographies, vol. 13, no. 1, pp. 1-20.

Pulugurtha, SS \& Kusam PR 2012, 'Modeling annual average daily traffic with integrated spatial data from multiple network buffer bandwidths', Transportation Research Records: Journal of the Transportation Research Board, vol. 2291, pp. 53-60.

Ross, Z, Ito, K, Johnson, S, Yee, M, Pezeshki, G, Clougherty, JE, Savitz, D \& Matte, T 2013, Spatial and temporal estimation of air pollutants in New York City: exposure assignment for use in a birth outcomes study, Environmental Health, vol. 12, no. 51.

Rosso, AL, Auchincloss, AH \& Michael, YL 2011, 'The urban built environment and mobility in older adults: a comprehensive review', Journal of Aging Research.

Rowles, GD 1981, 'The surveillance zone as meaningful space for the aged', The Gerontologist, vol. 21, no.3, pp. 304-311.

Rundle, A, Neckerman, KM, Freeman L, Lovasi, GS, Purcial, M, Richards, C, Sircar, N \& Weiss, C 2009, 'Neighbourhood food environment and walkability predict obesity in New York City', Environmental Health Perspectives, vol. 117, pp. 442-447.

Schaefer-McDaniel, N, Caughy, MOB, O'Campo, P \& Gearey, W 2010, 'Examining methodological details of neighbourhood observations and the relationship to health: a literature review', Social Science E Medicine, vol. 70, no. 2, pp. 277-292.

Shanaha, MJ \& Hofer, SM 2005, 'Social context in gene-environment interactions: Retrospect and prospect', The Journal of Gerontology Series B: Psychological Sciences and Social Sciences, vol. 60, pp. 65-760 .

Soja, EW 2010, Seeking spatial justice, University of Minnesota Press.

Stevens, A \& Coupe, P 1978, 'Distortions in judged spatial relations', Cognitive Psychology, vol. 10, pp. 422-437.

Thorndyke, PW \& Hayes-Roth, B 1982, 'Differences in spatial knowledge acquired from maps and navigation', Cognitive Psychology, vol. 14, pp. 560-589. 
Thorndyke, PW \& Stasz, C 1980, 'Individual differences in procedures for knowledge acquisition from maps', Cognitive Psychology, vol. 12, pp. 137-175.

Tolman, EC 1948, 'Cognitive maps in rats and men', Psychological Review, vol. 55, pp. 189.

Willis, KS, Holscher C, Wilbertz G \& Li, C 2009, 'A comparison of spatial knowledge acquisition with maps and mobile maps', Computers, Environment and Urban Systems, vol. 33, no. 2, pp. 100-110.

Yen, IH, Michael, YL \& Perdue, L 2009, 'Neighbourhood environment in studies of health of older adults: a systematic review', American Journal of Preventive Medicine, vol. 37, no. 5, pp. 455-463.

Zhou, C, Frankowski D, Ludford P, Shekhar, S \& Terveen, L 2007, 'Discovering personally meaningful places: an interactive clustering approach', ACM Transactions on Information Systems (TOIS), vol. 25, no. 12. 